\title{
Technique of Template Data Preparation for UAV Flight Mission Task
}

\author{
M.P. Mukhina \\ Aviation Computer-Aided Complexes Department \\ National Aviation University \\ Kyiv, Ukraine \\ m_mukhina@inbox.ru
}

\author{
I.V. Seden \\ Aviation Computer-Aided Complexes Department \\ National Aviation University \\ Kyiv, Ukraine \\ irochkaseden@gmail.com
}

\begin{abstract}
General technique of template data preparation is proposed for the tasks of visual correlation-extreme navigation, which includes the image and its descriptive representation. Descriptive representation is based on Speed-Up Robust Feature (SURF) method. The requirements and structure of database of unified template are developed including the integral index of fragment informativity. Experimental results show the stability of index and explicit minimum for informative fragment of image.
\end{abstract}

Keywords-template data; image features; correlation-extreme navigation; Speed-Up Robust Feature; descriptor covariance; integral index of informativity

\section{INTRODUCTION}

Application of technology of computer vision, including methods of image processing and image recognition theory is one of perspective branches of navigation and control systems development, which are able to define their location in space by means of comparing information, obtained from sensors of external information and reference information. Such systems define the concept of on-board highly-precise integrated navigation and control systems of UAV. Realization of requirements to highly precise detection of UAV location with correlation extreme navigation system is coupled with the quality and parameters of used reference images.

Correlation-extreme navigation system (CENS) is topical since it is an alternative of data fusion source of Inertial Navigation System (INS), instead of Satellite Navigation System (SNS). The principle of operation of CENS is based on a comparison of template realization of geophysical field (saved in computer memory) with the current realization obtained from the airborne sensor [1]. A degree of matching is represented in the form of correlation function, its extreme is found and is used to get positioning over map.

Accuracy of CENS positioning is determined by accuracy of airborne sensor and, of course, by accuracy of cartographic data. The high resolution of georefferencing images for visual CENS provides redundancy of information, requires large volume of memory to be allocated, needs high computational capability to be processed in real time, etc. Thus, the preliminary processing of georefferencing images is recommended with purpose of creation of unified template. It must contain minimal but sufficient data for reliable matching with heavy computational preprocessing

\section{Problem Statement}

\section{A. Detection of image informative areas}

To compare the images by the method of correlation analysis or to compare their specific features it is necessary to solve the problem of objects selection on the front plan in permanently unmoved ground. In case of using this principle aboard UAV the constant changing of objects and background takes place, so the detection of informative areas of image is needed. Consideration of small quantity of informative areas at the detection and recognition of objects decreases the requirements to computational costs of CENS realization aboard UAV. At the same time, during the process of elimination of non-informative areas, the areas containing objects may be excluded from the consideration. So, the problem of application of detection methods to find informativity of image areas and to select more informative areas for further consideration is present. Respectively [2] such problem is not solved nowadays at full degree.

The mathematical problem statement consists of the following. Let it be necessary to find the correspondence of some point $\left(x_{0}, y_{0}\right)$ of current image with reference one. Let's designate $f(x, y)$ as initial reference image. Consider some fragment of this image with center $\left(x_{0}, y_{0}\right)$ and size $(2 N+1) \times(2 N+1)$ pixels. Let's add informativity function of this fragment $I\left(x_{0}, y_{0}, N\right)$.

It is necessary to define:

- is this fragment informative;

- if it is not informative, is it possible to change its size to become informative.

To answer this question it is possible to use some criteria, based on comparison of informativity function $I$ with numerical threshold $T$. If $I \square T$, fragment is non-informative. In this case the size of fragment increases on some constant $N$ till the opposite condition is not fulfilled, or the size is not get some maximum value $N_{\max }$. 


\section{B. Representation of image specific features}

Image specific features can include feature points, lines (contours) and areas. The variety of methods are used to detect features and to compound descriptors which are unique for definite feature. The structure of descriptors may differ, however the basic principle used here can be easily expand for another method used to describe the image features. For further consideration, SURF descriptors are used [3].

The SURF detector is based on the determinant of the Hessian matrix:

$$
\mathbf{H}(x, y, \sigma)=\left|\begin{array}{ll}
L_{x x}(x, y, \sigma) & L_{y x}(x, y, \sigma) \\
L_{x y}(x, y, \sigma) & L_{y y}(x, y, \sigma)
\end{array}\right|,
$$

where $L_{x x}(x, y, \sigma)$ is convolution of the second order partial of Gaussian $\frac{\partial^{2}}{\partial x^{2}} g(\sigma)$ from the image in the point $(x, y)$. The same is for $L_{x y}(x, y, \sigma)$ and $L_{y y}(x, y, \sigma)$.

But for SURF the fast Hessian is found that is the approximation of matrix (1) by box filters. Dimension of filters is selected as $9 \times 9$ with scale $\sigma=1.2$ (minimal). The approximations are designated as $D_{x x}, D_{y y}, D_{x y}$. The weights are selected from Frobenius norm:

$$
\operatorname{det}\left(\mathbf{H}_{\text {approx }}\right)=D_{x x} D_{y y}-\left(0.9 D_{x y}\right)^{2} .
$$

In general case the descriptor of feature point by SURF method includes the following information: coordinates $P=\{x, y\}$, scale of Gaussian filter $M=\{\sigma\}$, gradient orientation $R=\{\alpha\}$, Laplacian $L=\{0,1\}$ (means either white spot on black background or black spot on white), and gradients of quadrants $D=\left\{D_{1}, D_{2}, \ldots, D_{64(128)}\right\}$, which surround the point.

The descriptor is calculated as the gradients for $4 \times 4=16$ quadrants around the feature point. Then each quadrant is divided further by 16 smaller quadrants. Four components on each quadrant must be computed that gives totally the 64 components of descriptor of area around the feature point. By the forming of descriptor array the values are weighted by Gaussian $3.3 \sigma$ and centered in the feature point to minimize the possible noise components.

The descriptor of feature point by SURF contains enough information to match the reference image and current one, but for reliable positioning some fields to the descriptor must be added and investigated.

\section{Analyses of existing methods of cartographic data preparation}

General requirements to unified template for CENS are formulated in [4], without any proposed structure and used methods of data processing. More detailed description of procedure of template data preparation and processing is given in [5]. The proposed presentation is based on technology of computer vision where the template is given as a scene. However, such representation has a significant drawback, namely the procedure of its formation cannot be fully formalized. And therefore it has no posibility to be fully automatized.

There are several methods of informativity determination.

Calculation of signal variance. More homogeneous the brightness signal is, the more area is informative.

Signal-to-noise ratio ( $S N R$ ). Using signal-to-noise ratio for determination of image area informativeness is suggested, more homogeneous area is, there will be less deviations of signal and, correspondently, less deviations of noise.

Correlation radius of signal. Correlation radius of signal determines the distance, where the readings of signal could be considered as statistically independent. More homogeneous the image area is, the greater radius of correlation will be.

Analysis of signal presence on image fragment. The method is based on analysis of optical wedge, which is divided on areas. For every area the function of average brightness and standard deviation are calculated. Then the getting of function of dependence of standard deviation of noise on brightness is realized. On the base of analysis of this function the conclusion about signal presence on the image could be made.

The considered approaches have the essential disadvantage: it is necessary to change iteratively (increase) the sizes of analyzed window for every point of image.

In this case, usage of multiple-scale introduction of images or images pyramid is the most effective. Images pyramid is a consequence of images, where the next image is obtained from previous one by means of filtering and decimation in two times.

\section{PRoposed TECHNiQUe TO PREPARE CARTOGRAPHIC DATA TEMPLATE}

Among computationally simple indexes of nonhomogeneity (uniqueness or, correspondently, informativity) of reference fragment is variance of image intensity, signal-tonoise ratio, correlation radius, etc [2], mentioned above.

Variance of image intensity inside the fragment is the index of fragment non-homogeneity and is determined by expression:

$$
\begin{aligned}
\sigma^{2}\left(x_{0}, y_{0}, N\right)= & \frac{1}{(2 N+1)^{2}} \sum_{x=-N}^{N} \sum_{y=-N}^{N}\left(f\left(x+x_{0}, y+y_{0}\right)\right)^{2} \\
& -\left(\frac{1}{(2 N+1)^{2}} \sum_{x=-N}^{N} \sum_{y=-N}^{N} f\left(x+x_{0}, y+y_{0}\right)\right)^{2},
\end{aligned}
$$

where $x_{0}, y_{0}$ are central points of image, for which the informativity is defined, $N$ is the chosen size of area of point $x_{0}, y_{0}$. 
Such indexes are used for correlation approach to compare current and reference images. But many researcheres, especially [6], define separately correlation-extreme methods of images comparison and methods of comparison of images characteristic features. Further it will be shown, that even the representation of image by characteristic features allows applying conventional methods of correlation-extreme analysis with minimal changes, and in such way, to extend the term of correlation extreme systems.

Research was done on the example of one of indexes of informativity by classic definition of correlation methods, particularly, variance (2). If the image is represented as a structure by descriptive features of special points type, found by SURF method, then instead of informative feature - image intensity, that is used in formula (2), the descriptors of point, which are represented in the mentioned above form, will be used.

On intuitive level it is clear, that high quantity of feature points, found in fragment, corresponds to better informativity, and correspondingly their uniqueness and distinctness one from another will provide higher reliability of comparison. Let's estimate the uniqueness through covariance of correspondent pairs of feature points on the fragment. Since the descriptors of points are centered and not displaced with final second moment, then the expression of covariance of pairs of random descriptors would be:

$$
\operatorname{cov}\left(D_{i}, D_{j}\right)=M\left[D_{i} D_{j}\right]
$$

Let's take into account that descriptors are vector values, particularly, $[8 \times 8]$ or $[64 \times 1]$, and then expression (3) will be:

$$
\operatorname{cov}\left(D_{i}, D_{j}\right)=M\left[D_{i}^{T} D_{j}\right]
$$

Correspondingly, covariance value (4) for pair of random descriptors will be scalar, if the descriptors are written as vectors $[64 \times 1]$. Then covariance by pairs of all descriptors of image fragment can be calculated in single operation of matrix product

$$
\begin{aligned}
\operatorname{cov}(D, D) & =M\left[D^{T} D\right] \\
& =M[[m \times 64] \times[64 \times m]]=M[[m \times m]],
\end{aligned}
$$

where $m$ is a number of found descriptors at the fragment. So, formula of covariance for representation of fragment characteristic features as SURF points, will be the following:

$$
\begin{aligned}
& \operatorname{cov}(D, D)=K=M\left[\mathbf{D}^{\mathrm{T}} \cdot \mathbf{D}\right] \\
& =\frac{1}{m^{2}}\left[\begin{array}{ccc}
D_{1}(1) & \ldots & D_{1}(m) \\
\vdots & \ddots & \vdots \\
D_{64}(1) & \cdots & D_{64}(m)
\end{array}\right]^{\mathrm{T}} \cdot\left[\begin{array}{ccc}
D_{1}^{\prime}(1) & \ldots & D_{1}^{\prime}(m) \\
\vdots & \ddots & \vdots \\
D_{64}^{\prime}(1) & \cdots & D_{64}^{\prime}(m)
\end{array}\right] .
\end{aligned}
$$

The greater the difference between descriptors is, the smaller the value of covariance (5) will be. It is also necessary to mention that descriptor matrix is already normalized due to peculiarities of SURF method.

\section{EXPERIMENTAL RESEARCHES OF IMAGE FRAGMENTS INFORMATIVITY}

To research the proposed technique of informativity estimation and reliability of image matching two image fragments (Fig. 1) were taken with coordinates in points A1 and $\mathrm{A} 2$, which correspond to region of low informativity (frequently mentioned as texture features) and to region with a number of artificial objects, which are distinctly observed from the background.

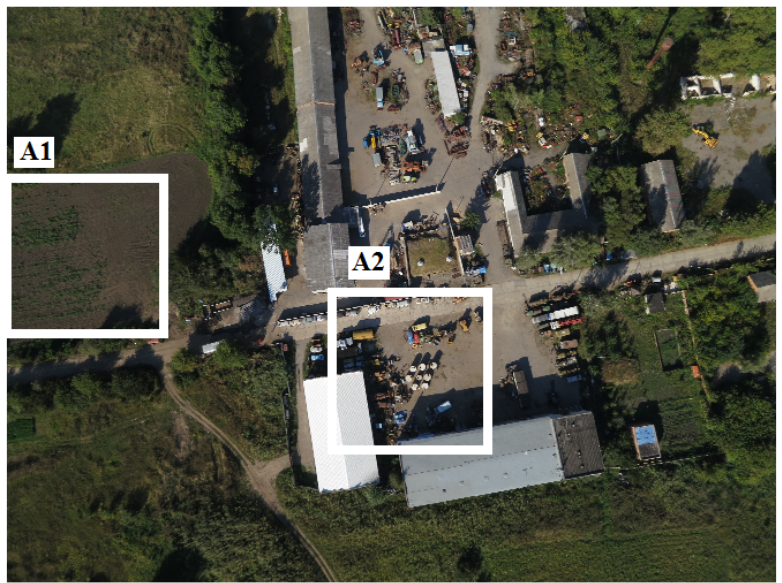

Fig. 1. Meta template image with size $3000 \times 4000$ pixels and with two initial points of fragment, maximum size of which $(800 \times 800)$ is shown by white contour.

Choosing points A1 and A2 as left upper corner for area of image fragment with corresponding sizes $100 \times 100,200 \times 200$, $300 \times 300,400 \times 400,500 \times 500,600 \times 600,700 \times 700,800 \times 800$, for obtained fragments the indexes of informativity according to both approaches were calculated: image intensity variance (2) for classic correlation analyses and covariance (5) for descriptive representation of fragment. Graphs of dependences of informativity indexes $K$ and $\sigma$ on fragment size are shown in Figs 2, 3.

Increasing the size of the first fragment with mostly texture features does not cause corresponding decreasing of covariance value $K$. The number of descriptors becomes greater, but they are similar to each other, because they correspond to texture peculiarities and are repeated. According to such informativity index as variance $\sigma$ for the first fragment it is not possible to solve unambiguously whether the fragment of given sixe is informative or not and to determine optimal size of template.

For the second fragment the dependence of covariance $K$ on the fragment size has explicit minimum (for size $200 \times 200$ ), that corresponds to maximum value of informativity and variety of descriptors. The value of variance $\sigma$ for this size is also maximum, but hereafter it decreases and reaches the second maximum, that may cause variety in solution. 
In such way $\square$, for estimation of fragment informativity $\square$ the where $\sigma, \rho_{k}, \mathrm{SNR}, K$ are variance, correlation radius, signalintegral index $H$ is proposed to use. It consists of the indexes of classic correlation approach, such as variance, signal-tonoise ratio, correlation radius, and index of correlationdescriptive approach, that includes the index of covariance of characteristic features.

$$
H=w_{\sigma} \sigma+w_{\rho_{k}} \rho_{k}+w_{\mathrm{SNR}} \mathrm{SNR}+w_{K} K,
$$
to-noise ratio, covariance index; $w_{\sigma}, w_{\mathrm{Pk}}, w_{\mathrm{SNR}}, w_{K}$ are weight coefficients, which correspond to the selected influence of given index on the integral index, and can be changed according to requirements of cartographic template, particularly $\square$ for covariance indexthe weight coefficient must be negative.
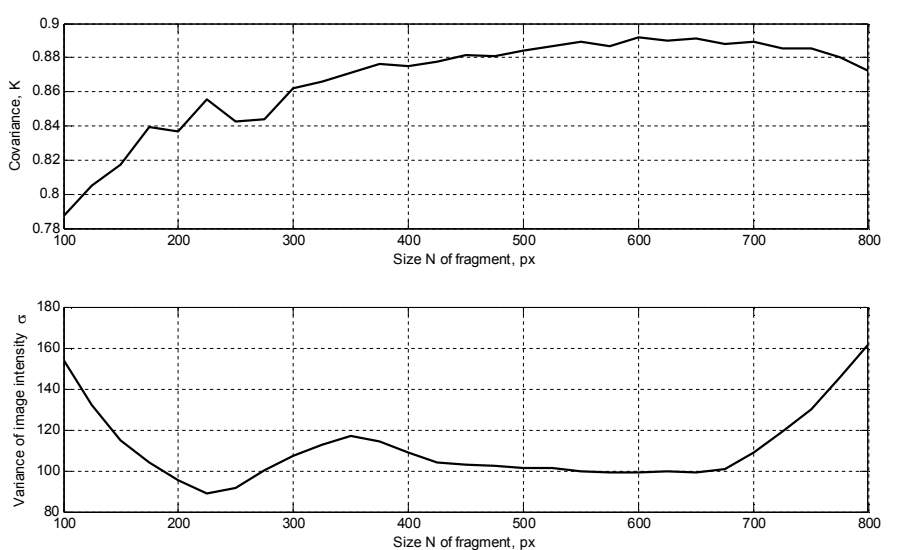

$\mathrm{y} \square$ ig. 2. Size of the first (non informative) fragment is varied from $100 \times 100$ till $800 \times 800$ pixels with fixed left upper point.
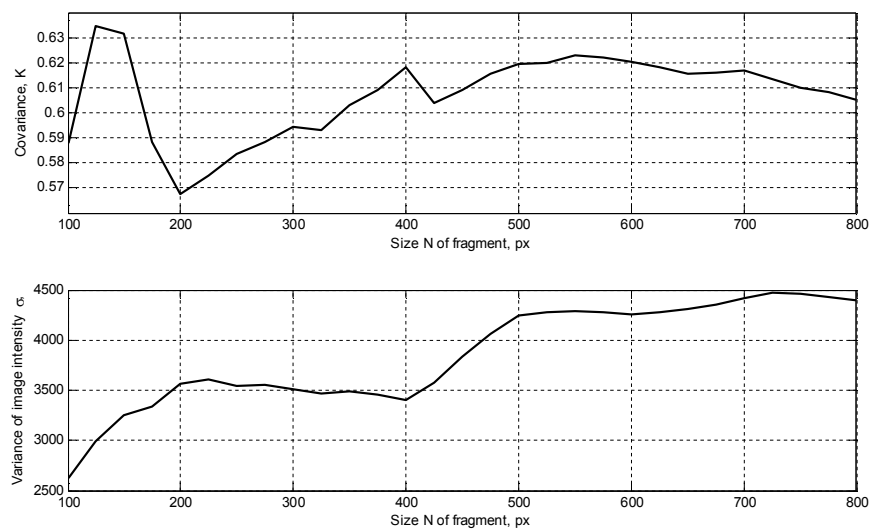

$\mathrm{y} \square$ ig. 3. Size of the second fragment (with high number of artificial objects) is varied from $100 \times 100$ till $800 \times 800$ pixels with fixed left upper point.

\section{CONCLUSIONS}

General structure of unified template representation is proposed for the tasks of visual correlation-extreme navigation, which includes the image and its descriptive representation. Size of template fragment and its major representation is proposed to be estimated according to integral index of informativity $\square$ with unification of classic correlation approach and descriptive one, for which the index of descriptors covariance is determined. Its calculation needs only $\square$ operation of descriptors matrix product, and results of researches show the stability $\square$ of index and explicit minimum for informative fragment of image.

$$
\mathrm{Ry} \square \mathrm{y} \square \mathrm{y} \square \mathrm{Ry} \square \mathrm{NCy} \square \mathrm{S}
$$

[1] M.P. Mukhina and I.V. Seden, “Analy $\square$ sis of modern correlation extreme navigation sy $\square$ stem $\$$ y $\square \square$ lectronics and Control Sy $\square$ stenmol. 1 (39), 2014, pp. $95-101$.

[2] M.N. Krasilshchikov and G.G. Serebriakov, Control and guidance of unmanned manuevered aerial vehicles on the basis of modern infromation technologies, Moscow, y $\square$ IZMATLIT preş2003, $263 \mathrm{p}$.

[3] H. Bay $\square$, T. Tuy $\square$ telaarrsd L. Van Gool. SURy: Speeded up robust features. $\mathrm{y} \square$ uropean Conference on Computer Visionyol. 1, pp.404-417, 2006.

[4] V.K. Baklitskii, Correlation extreme methods of navigation and guidance, Tver', "Knizhny $\square$ i klübpress, 2009, 360 p.

[5] K.K. Veremeenko and others Modern information technologies in the problems of navigation and control of unmanned manuevered aerial vehicles, Moscow, $\square \square$ IZMTLIT press, 2009, $556 \mathrm{p}$.

[6] M.V. Medvedev and T.A. Sinichkina, "Objects classification on the basis of data taken from images". Information technologies in economics, education and bussiness, Proceedings of V International science practical conference, Saratov, $\mathrm{y} \square$ ebruary $\square 201$ p, f4-65. 